2020-10-13

\title{
Investigation of Surrogate Biomarkers Associated with Macular Pigment Status in a Group of Older Irish Adults
}

\author{
Grainne Scanlon \\ Technological University Dublin, grainne.scanlon@tudublin.ie \\ John S. Butler \\ Technological University Dublin, john.s.butler@tudublin.ie \\ Daniel McCartney \\ Technological University Dublin, daniel.mccartney@tudublin.ie
}

See next page for additional authors

Follow this and additional works at: https://arrow.tudublin.ie/scschphyart

Part of the Dietetics and Clinical Nutrition Commons, and the Optometry Commons

\section{Recommended Citation}

Scanlon, G., Butler, J.S., McCartney, D, Loskutova, E. \& Kenny, RA \& Loughman J. (2020). Investigation of surrogate biomarkers associated with macular pigment status in a group of older adults. Optometry \& Vision Science97(10), pp.79-888 doi:10.1097/OPX.0000000000001587

This Article is brought to you for free and open access by the School of Physics \& Clinical \& Optometric Science at ARROW@TU Dublin. It has been accepted for inclusion in Articles by an authorized administrator of ARROW@TU Dublin. For more information, please contact arrow.admin@tudublin.ie, aisling.coyne@tudublin.ie, gerard.connolly@tudublin.ie.

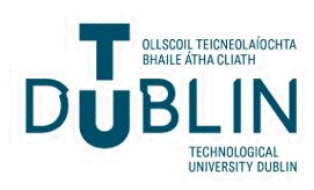




\section{Authors}

Grainne Scanlon, John S. Butler, Daniel McCartney, Ekaterina Lostutova, Rose Anne Kenny, and James Loughman 


\title{
Investigation of Surrogate Biomarkers Associated with Macular Pigment Status in a Group of Older Irish Adults
}

\author{
Grainne Scanlon, MPhil, PhD, ${ }^{1 *}$ John S. Butler, $\mathrm{PhD},{ }^{2}$ Daniel McCartney, PhD, ${ }^{3}$ Ekaterina Loskutova, $\mathrm{PhD},{ }^{1}$ \\ Rose A. Kenny, MD, PhD, ${ }^{4,5,6}$ and James Loughman, $\mathrm{PhD}^{1,7}$
}

\begin{abstract}
SIGNIFICANCE: Macular pigment (MP) confers potent antioxidant and anti-inflammatory effects at the macula; however, its optical density in the eye is not routinely measured in clinical practice.

PURPOSE: This study explored a range of surrogate biomarkers including anthropometric, clinical, and plasma measures that may be associated with lower MP optical density (MPOD).

METHODS: Two thousand five hundred ninety-four subjects completed a full MP assessment as part of wave 1 of The Irish Longitudinal Study of Aging. Macular pigment optical density was measured using customized heterochromatic flicker photometry. Clinical (blood pressure), plasma (lipoproteins, inflammatory markers), and anthropometric (waist, hip, height, weight) biomarkers were measured for each participant.
\end{abstract}

RESULTS: Mean (standard deviation) MPOD for the study group was $0.223(0.161)$, with a range of 0 to 1.08 . One-way ANOVA revealed that MPOD was significantly lower among participants with low plasma high-density lipoprotein (HDL; $P=.04)$, raised plasma triglyceride-to-HDL ratio $(P=.003)$, and raised total cholesterol-to-HDL ratio $(P=.03)$. Subjects with an elevated waist circumference $(W C)$ had a significantly lower MPOD (mean, 0.216 [0.159]) compared with those with an ideal WC (mean, 0.229 [0.162]; $P=.03$ ). Significant correlates of MPOD on mixed linear model analysis included education, smoking status, and WC.

CONCLUSIONS: Higher abdominal fat is associated with lower MPOD in this representative sample of older Irish adults. Although altered lipoprotein profiles (low HDL, raised triglyceride-to-HDL ratio, raised total cholesterol-to$\mathrm{HDL}$ ratio) may affect the transport, uptake, and stabilization of carotenoids in the retina, these plasma biomarkers were not predictive of low MPOD after adjustment for abdominal circumference. Although WC emerged as a viable anthropometric predictor of lower MPOD, its effect size seems to be small.

Optom Vis Sci 2020:00:00-00. doi:10.1097/OPX.0000000000001587

Copyright () 2020 American Academy of Optometry

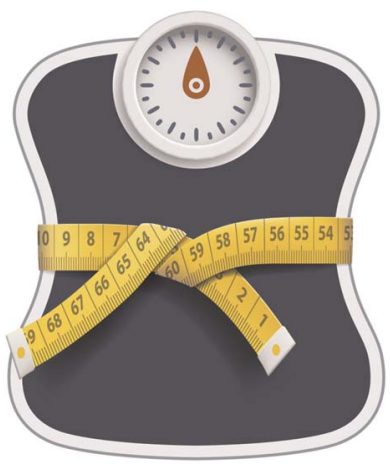

\begin{abstract}
Author Affiliations:
${ }^{1}$ Centre for Eye Research Ireland, School of Physics, Clinical and Optometric Sciences, Environmental Sustainability and Health Institute, Technological University Dublin, City Campus, Dublin, Ireland

${ }^{2}$ School of Mathematical Sciences, Technological University Dublin, City Campus, Dublin, Ireland

${ }^{3}$ School of Biological and Health Sciences, Technological University Dublin, City Campus, Dublin, Ireland ${ }^{4}$ The Irish Longitudinal Study on Ageing, Trinity College Dublin, Dublin, Ireland ${ }^{5}$ Mercer's Institute for Successful Ageing, St. James' Hospital, Dublin, Ireland

${ }^{6}$ Department of Medical Gerontology, Trinity College Dublin, Dublin, Ireland

${ }^{7}$ African Vision Research Institute, University of KwaZulu-Natal, Durban, South Africa

*grainne.scanlon@tudublin.ie
\end{abstract}

The concept that macular pigment may have a protective role in neurodegenerative eye conditions such as AMD, glaucoma, and diabetes is well documented. ${ }^{1-3}$ The macula is particularly important for central high-resolution vision and blindness results when this area is lost to disease. The carotenoids lutein, zeaxanthin, and meso-zeaxanthin, collectively known as macular pigment, accumulate at the macula to the exclusion of all other dietary carotenoids. Macular pigment has a unique distribution within the retina, selectively located within the fibers of Henle in the fovea and in the inner nuclear layer of the parafovea, ${ }^{4}$ implying that macular pigment plays an important role in vision and macular health and that it is biologically relevant to the eye. ${ }^{5,6}$

The selective absorption of short-wavelength light before photoreceptor light capture means that macular pigment plays an important role in visual performance in healthy eyes. ${ }^{7}$ Concomitantly, through its optical filtration and antioxidant properties, macular pigment protects the retina from (photo)-oxidative damage, thereby potentially reducing the risk of various eye diseases including AMD $^{8}$ and diabetes. ${ }^{9,10}$ Decreased macular pigment optical density seems to be a risk factor for the development of these 
diseases, ${ }^{11-13}$ and numerous studies investigating the effects of macular pigment augmentation have also reported beneficial effects in diseased eyes. ${ }^{8,9}$ Although the etiopathogenesis of conditions such as AMD and diabetes remains a matter of debate, there is growing consensus that oxidative damage ${ }^{14,15}$ and associated inflammation ${ }^{16,17}$ play a significant role. The macular carotenoids exhibit neuroprotective functions at the macula, ${ }^{2,18}$ and it has been shown that lutein and zeaxanthin can affect immune responses, reduce inflammation, and have antiangiogenic properties in the eye. ${ }^{2,19,20}$ Therefore, the density of macular pigment offers potential as a clinical biomarker of retinal health.

Macular pigment levels can be measured in vivo, but the relevant instrumentation is not commonly available in clinical practice. Despite some attempts, there is still limited uptake of instruments that can successfully measure macular pigment optical density in vivo, in clinical practice. ${ }^{21}$ Other indirect techniques for assessing macular pigment status such as dietary and plasma analysis have limited practical application and are generally used only in research settings. Previous investigators have examined and identified possible predictors of macular pigment optical density and found associations with sex, ${ }^{22}$ iris color, ${ }^{23}$ dietary intake, ${ }^{24-26}$ plasma cholesterol and lipoprotein status, ${ }^{25,27,28}$ metabolic status, ${ }^{12,13}$ overweight/obesity status, ${ }^{29-31}$ and smoking. ${ }^{29}$ Many of these studies, however, were based on opportunistic sampling and restricted to small sample sizes ${ }^{12,13,22,23,26,28,31}$ and younger age groups ${ }^{25,30}$ or relied on self-reported data. ${ }^{29}$ Although one study investigated determinants of macular pigment optical density on a larger older cohort $(n=1698)$, this research was conducted on female subjects only. ${ }^{24}$

The evidence that does exist, however, suggests that these relationships are worth exploring in more detail. Metabolic comorbidities including overweight/obesity, ${ }^{29,30}$ hypertension, ${ }^{29}$ and dyslipidemia, ${ }^{25}$ may adversely affect macular pigment by compromising the availability, ${ }^{31}$ transport, ${ }^{27}$ and assimilation ${ }^{27}$ of dietary carotenoids in the retina. Consequently, there is merit in exploring the association between macular pigment optical density and these more commonly measured clinical and biometric parameters, which might be used to identify patients at risk of low macular pigment, so that practitioners can implement strategies for preventative intervention. The capacity of such an alternate biomarker that is more readily and routinely measured (i.e., blood pressure, plasma lipoproteins, abdominal fat) to expedite the identification of patients at risk of low macular pigment could be particularly important and may be used to counsel patients. It is important to recognize that to minimize the risk of macular pigment depletion it is not sufficient to supplement with macular carotenoids, but rather to suggest that these proxy biomarkers may be used to help individuals (i.e., older adults free of ocular pathology) to take a more holistic evaluation of their diet and lifestyle concerning macular pigment optical density. Management guidance may be given to patients, reinforcing the need to incorporate brightly colored fruit and vegetables as part of one's diet, as they are rich in a broad range of antioxidants including the target carotenoids lutein and zeaxanthin. This study was designed therefore to address some of the limitations of previous investigations by examining the predictive capacity of a range of measured (rather than self-reported) clinical (blood pressure), plasma (lipoproteins, inflammatory markers), and anthropometric (abdominal fat) parameters as possible predictors of macular pigment optical density in a large representative group $(n=2594)$ of older men and women.

\section{METHODS}

\section{Study Design and Population}

Cross-sectional data from wave 1 of The Irish Longitudinal Study on Aging were analyzed in this study, which was conducted during the period between October 2009 and July 2011. Participants were recruited to provide a stratified clustered sample representative of the population 50 years or older living in Ireland as described in detail elsewhere. ${ }^{32,33}$ In brief, information on health behaviors and lifestyle patterns was captured by trained professional interviewers in participants' own homes using computer-aided personal interview. ${ }^{34}$ Participants were then invited to take part in a health assessment. ${ }^{35}$ Macular pigment measurement was conducted only on participants who attended a health center (Fig. 1). The current study was approved by the Technological University Dublin Research Ethics Committee, and all experimental procedures adhered to the tenets of the Declaration of Helsinki. All participants provided written informed consent before participation in the study.

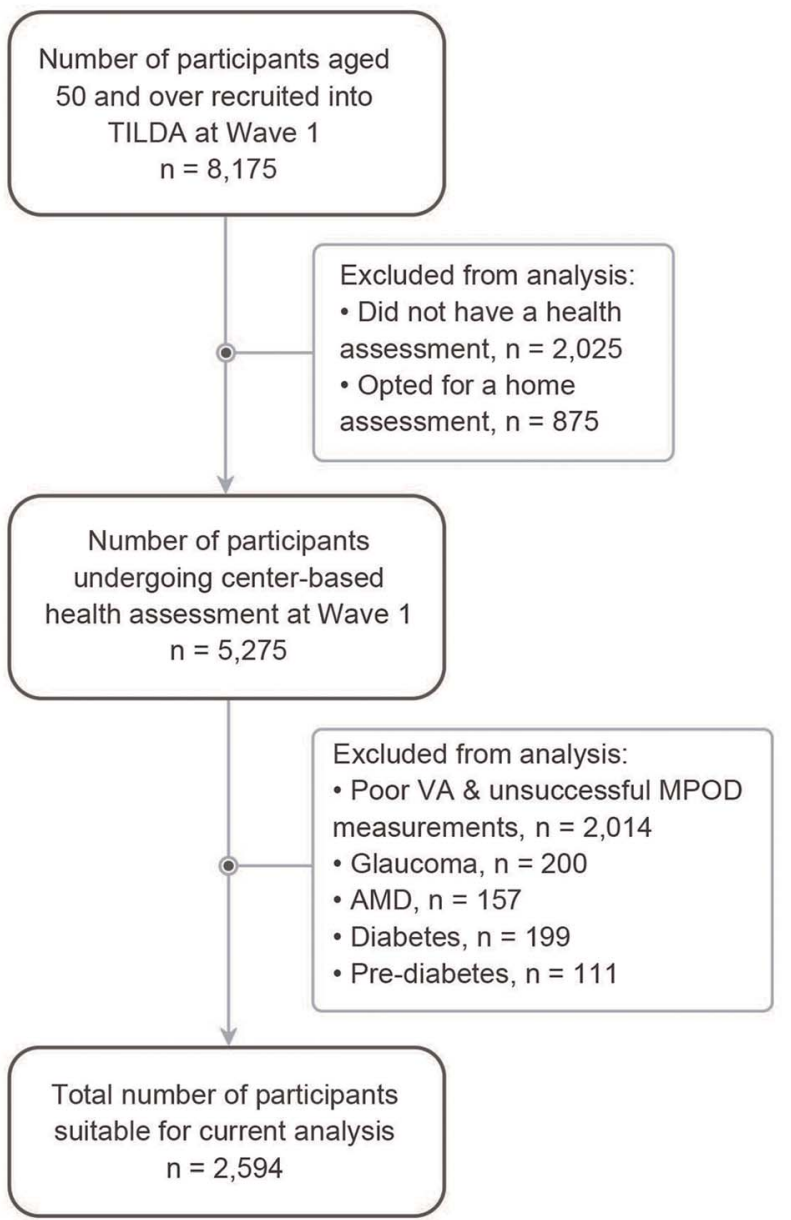

FIGURE 1. Flowchart illustrating the selection of study participants and reasons for exclusion from the original participant group. MPOD = macular pigment optical density; TILDA = The Irish Longitudinal Study on Aging; VA = visual acuity. 


\section{Demographic, Health, and Lifestyle Factors}

Demographic and lifestyle factors were captured for the study group. The presence or absence of hypertension was also examined. Participants were defined as hypertensive if mean seated systolic blood pressure exceeded $140 \mathrm{mmHg}$ or mean seated diastolic blood pressure exceeded $90 \mathrm{mmHg}$.

\section{Macular Pigment Optical Density Assessment}

Macular pigment optical density was measured by customized heterochromatic flicker photometry using the Macular Metrics Densitometer (Macular Metrics, Rehoboth, MA). This device was modified specifically for The Irish Iongitudinal Study on Aging, and the method of measurement has been described in detail elsewhere. ${ }^{36}$ In brief, macular pigment optical density was measured centrally at $0.5^{\circ}$ (i.e., $1^{\circ}$ stimulus) and peripherally at $7^{\circ}$ (parafoveal target at $7^{\circ}$ ). Macular pigment assessment was carried out on the eye with the best visual acuity, or if there was equal vision in both eyes, the right eye was chosen. ${ }^{36}$ Corrected visual acuity was measured using the Early Treatment Diabetic Retinopathy Study LogMAR chart at a distance of $4 \mathrm{~m}$ using participants' existing prescription where necessary. Participants with a visual acuity worse than 0.5 LogMAR were excluded from the analysis (Fig. 1).

\section{Anthropometric Assessment}

Height (in centimeters) and weight (in kilograms) were measured to one decimal place using a Seca wall-mounted measuring rod and Seca electronic floor scales, as described in detail elsewhere. ${ }^{34}$ Body mass index was calculated from measured height and weight as weight (in kilograms)/height (in meter squared), with obesity classified as a body mass index $>30 \mathrm{~kg} / \mathrm{m}^{2}$. ${ }^{37}$ Waist and hip circumferences were measured to the nearest centimeter. Ideal waist circumference was defined as a waist circumference of $\leq 88$ $\mathrm{cm}$ in women and $\leq 102 \mathrm{~cm}$ in men, whereas central obesity was defined as a waist circumference $>88 \mathrm{~cm}$ in women and $>102$ $\mathrm{cm}$ in men. Waist-to-height ratio and waist-to-hip ratio were calculated based on measured data. Cutoff thresholds for ideal and excess waist-to-height ratio were taken as less than or greater than 0.53 in women and 0.57 in men, respectively. For waist-to-hip ratio, cutoff thresholds of less than or greater than 0.85 in women and less than or greater than 1.00 in men defined ideal and elevated waist-to-hip ratios. ${ }^{38,39}$

\section{Plasma Analysis}

A blood sample was provided for plasma analysis using defined phlebotomy protocols, which are described in detail elsewhere. ${ }^{34,35}$ Respondents were not asked to fast before the health assessment, and plasma was analyzed for a complete lipid profile, including total cholesterol, high-density lipoprotein, low-density lipoprotein, and triglycerides, each measured in millimoles per liter. Triglycerideto-high-density lipoprotein ratio, total cholesterol-to-high-density lipoprotein ratio, and non-high-density lipoprotein cholesterol (total cholesterol minus high-density lipoprotein) were calculated from measured data. Cutoffs were applied to indicate high/low or ideal plasma lipid levels as per 2016 European Society of Cardiology/ European Atherosclerosis Society guidelines (Table 2). ${ }^{40}$ Plasma vitamin D levels (25-hydroxyvitamin D; in nanomoles per liter) were also measured, and participants were considered vitamin $D$ deficient if plasma levels were $\leq 50 \mathrm{nmol} / \mathrm{L}$ and sufficient if plasma levels were $>50 \mathrm{nmol} / \mathrm{L}$ as per Institute of Medicine vitamin D guidelines. ${ }^{41} \mathrm{In}$ flammatory marker C-reactive protein (in micrograms per liter) and glycated hemoglobin (in percent) were also measured. Threshold values of $>3.00 \mu \mathrm{g} / \mathrm{L}$ for high and $\leq 3.00 \mu \mathrm{g} / \mathrm{L}$ for ideal were used for plasma C-reactive protein, ${ }^{42}$ and cutoff values of $\leq 5.00 \%$ and $>5.00 \%$ were used for glycated hemoglobin.

\section{Statistical Analysis}

The statistical software package SPSS for Microsoft Windows (v. 25.0; IBM Corp., Armonk, NY) was used for analysis. To account for the fact that the study response rate varied between different subgroups of the population, inverse probability weights were calculated for the main sample (computer-assisted personal interview participants) using the Quarterly National Household Survey (2010). The probability of participation in the health center also varied according to health, education, age, and smoking; therefore, a specific "health center weight" was created. A detailed description of the weighting procedures used in The Irish Longitudinal Study on Aging is given elsewhere. ${ }^{43}$

Demographic data for eligible study participants were compared with those of the overall health center population using $x^{2}$ analyses, to explore if they differed from the representative sample. Data for the study group were tested for normality using the Kolmogorov-Smirnov test. One-way ANOVA was used to test differences in means for normally distributed parameters. For categorical data, cross-tabulation with $\mathrm{x}^{2}$ analysis was used. Pearson product-moment correlation tests were performed to assess the relationship between normalized macular pigment optical density and other study variables, where appropriate. A mixed linear model analysis (fixed effect) was carried out to estimate the independent association between putative predictor variables (behavioral, clinical, anthropometric, and plasma biomarker parameters) and normalized macular pigment optical density. Data are presented as mean (standard deviation) throughout. The level of statistical significance was set at $P<.05$ for all analyses.

\section{RESULTS}

In total, 8175 individuals 50 years or older participated in the study. Of these, 5275 consented to and participated in the health center-based assessment as part of wave 1 of The Irish Longitudinal Study on Aging. For various reasons, 2681 participants were excluded from the current analysis, leaving an overall study population of 2594 (Fig. 1). The main reasons for exclusion related to unsuccessful macular pigment optical density measurement or the presence of retinal pathology. Participants were identified as having AMD, cataracts, and glaucoma from the computer-assisted personal interview. The presence or absence of diabetes was identified from prescribed diabetes medications at the time of the interview (identified using the Anatomic Therapeutic Classification codes "A10A" for insulin and "A10B" for oral hypoglycemic medications), from the computer-assisted personal interview, and/or from measured glycated hemoglobin (glycated hemoglobin, $>6.4 \%$ [diabetes] and 5.7 to $6.4 \%$ [pre-diabetes]), as per American Diabetes Association cutoff values. ${ }^{44}$

$A x^{2}$ test of independence was performed to examine the relationship between the center population $(n=5275)$ and participants with a valid macular pigment optical density assessment $(n=2594)$. The age profile of study participants (mean age, 62.0 [8.5] years; range, 50 to 93 years) was similar to the overall health center-assessed population (mean age, 61.4 [7.6] years; range, 51 to 86 years), and there was no significant difference in sex or education status between the two groups ( $P=.88$ and $P=.77$, respectively). 
The distribution of macular pigment optical density was skewed; therefore, a square root transformation of the macular pigment optical density data was performed. The derived data were normally distributed and used as the dependent variable for subsequent statistical analyses of macular pigment optical density. For ease of interpretation, mean and standard deviation of macular pigment optical density data are presented as the nontransformed original measure for participants. Mean (standard deviation) macular pigment optical density for the study group was $0.223(0.161)$, with a range of 0 to 1.08. Differences in normalized macular pigment optical density by demographic (age group, sex, education status), health, and lifestyle variables (smoking, hypertension, physical activity, and cataracts) were examined. Our findings concur with previously reported statistically significant associations between sex, educational status, and smoking with macular pigment optical density in The Irish Longitudinal Study on Aging data set $(P<.05$ for all), despite a slightly different study sample (participants with AMD, glaucoma, diabetes, and pre-diabetes were excluded from the current analysis of healthy individuals but not in a previous study). ${ }^{29}$

\section{Clinical, Anthropometric, and Biomarker Associations with MPOD}

\section{Clinical Factors}

We found no correlation between measured hypertension status (with or without treatment) and macular pigment optical density in the current study ( $\mathrm{n}=2591 ; t=1.105 ; P=.33$ ).

\section{Anthropometric Factors}

Macular pigment optical density was significantly and negatively correlated with all anthropometric measures (body mass index, waist circumference, waist-to-height ratio, waist-to-hip ratio) on univariate analysis (Pearson $r=-0.047,-0.073,-0.060$, and -0.081 , respectively; $P<.05$ for all). Waist circumference, however, emerged as the only anthropometric variable associated with lower macular pigment optical density on binary univariate analysis. Participants with an elevated waist circumference had a significantly lower macular pigment optical density (mean, 0.216 [0.159]) compared with participants with ideal waist circumference (mean, 0.229 [0.162]; $\mathrm{n}=2589 ; t=2.119 ; P=.03$; Table 1, Fig. 2). The violin plots in Fig. 2 show similar distribution density plots and interquartile ranges for participants with excess and ideal waist circumference. Although the mean macular pigment optical density was significantly lower among subjects with a higher waist circumference, the differences were subtle and not clinically meaningful (value, 0.013 optical density units), as illustrated in Fig. 2. Macular pigment optical density did not differ significantly according to any other anthropometric measures ( $P>.05$ for all; Table 1$)$.

\section{Plasma Biomarkers}

Participants with ideal plasma levels of high-density lipoprotein had a significantly higher macular pigment optical density than did those with low high-density lipoprotein levels ( $n=2587$; $t=-2.077 ; P=.04$; Table 2, Fig. 3A). Macular pigment optical density was also significantly lower among participants with a raised triglyceride-to-high-density lipoprotein ratio ( $n=2587$; $t=2.994, P=.003$; Table 2, Fig. 3B) and among participants with a raised total cholesterol-to-high-density lipoprotein ratio ( $n=2574 ; t=2.166 ; P=.03$; Table 2, Fig. $3 C$ ). The violin plots in Fig. 3 show similar distribution plots and interquartile ranges for each of the three groups. The magnitude of all statistically significant differences $(0.011,0.019$, and 0.015 optical density units, respectively) was not, however, clinically important, as illustrated in Fig. 3. One-way ANOVA revealed no significant difference in macular pigment optical density levels for any other plasma biomarkers ( $P>.05$ for all; Table 2$)$.

TABLE 1. MPOD according to anthropometric biomarkers in the study population (normalized MPOD)

\begin{tabular}{|c|c|c|c|c|c|c|}
\hline Variable & n & Mean (SD) MPOD & 25th & 50th & 75th & $P$ sig. \\
\hline \multicolumn{7}{|l|}{ Anthropometric biomarkers } \\
\hline \multicolumn{7}{|l|}{$\mathrm{BMI}\left(\mathrm{kg} / \mathrm{m}^{2}\right)$} \\
\hline Ideal $(\leq 30)$ & 1387 & $0.223(0.161)$ & 0.100 & 0.191 & 0.310 & .83 \\
\hline Excess $(>30)$ & 1203 & $0.223(0.160)$ & 0.101 & 0.195 & 0.316 & \\
\hline \multicolumn{7}{|l|}{ WC (cm) } \\
\hline Ideal ( $\leq 102 \mathrm{M}, \leq 88 \mathrm{~F})$ & 1433 & $0.229(0.162)$ & 0.105 & 0.196 & 0.319 & $.03^{*}$ \\
\hline Excess (>102 M, >88 F) & 1158 & $0.216(0.159)$ & 0.096 & 0.187 & 0.301 & \\
\hline \multicolumn{7}{|l|}{ WHpR } \\
\hline Ideal ( $\leq 1.00 \mathrm{M}, \leq 0.85 \mathrm{~F})$ & 1637 & $0.225(0.158)$ & 0.105 & 0.195 & 0.314 & .13 \\
\hline Excess $(>1.00 \mathrm{M},>0.85 \mathrm{~F})$ & 954 & $0.219(0.165)$ & 0.093 & 0.189 & 0.307 & \\
\hline \multicolumn{7}{|l|}{ WHtR } \\
\hline Ideal $(\leq 0.57 \mathrm{M}, \leq 0.53 \mathrm{~F})$ & 1307 & $0.228(0.161)$ & 0.107 & 0.197 & 0.317 & .08 \\
\hline Excess $(>0.57 \mathrm{M},>0.53 \mathrm{~F})$ & 1282 & $0.219(0.162)$ & 0.095 & 0.188 & 0.306 & \\
\hline \multicolumn{7}{|c|}{$\begin{array}{l}\text { ANOVA was used to check for MPOD differences among anthropometric and physical biomarkers. } P \text { values are reported using MPOD square root trans- } \\
\text { formation (normalized MPOD) and reflect the probability associated with the given F statistic. The following cutoffs were applied to indicate ideal or excess } \\
\text { obesity measures for the following variables: BMI (ideal, } \leq 30 \text {; excess, }>30 \mathrm{~kg} / \mathrm{m}^{2}, W \mathrm{WC} \text { (ideal, } \leq 88 \mathrm{~cm} \mathrm{~F} \text { and } \leq 102 \mathrm{~cm} \mathrm{M;} \mathrm{excess,}>88 \mathrm{~cm} \mathrm{~F} \text { and }>102 \mathrm{~cm} \mathrm{M} \text { ), } \\
\text { WHtR (ideal, } \leq 0.53 \mathrm{~F} \text { and } \leq 0.57 \mathrm{M} \text {; excess, }>0.53 \mathrm{~F} \text { and }>0.57 \mathrm{M} \text { ), and WHpR (ideal } \leq 0.85 \mathrm{~F} \text { and } \leq 1.00 \mathrm{M} \text {; excess, }>0.85 \mathrm{~F} \text { and }>1.00 \mathrm{M}{ }^{37-39} \text { ). * Sig- } \\
\text { nificant at the } .05 \text { level. BMI = body mass index; F = female; } \mathrm{M}=\text { male; MPOD = macular pigment optical density; SD = standard deviation; sig. = } \\
\text { significance; WC = waist circumference; WHpR = waist-to-hip ratio; WHtR = waist-to-height ratio. }\end{array}$} \\
\hline
\end{tabular}




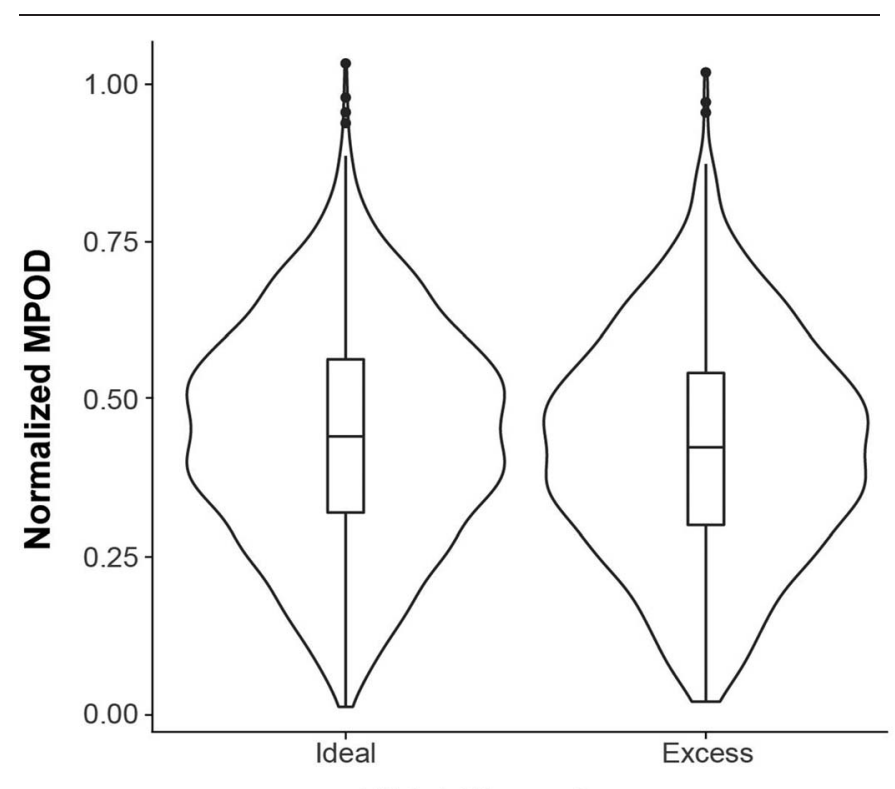

Waist Circumference

FIGURE 2. Violin plot of MPOD distribution according to waist circumference status, illustrating the substantially lower MP levels among subjects with a higher waist circumference (ANOVA). MP = macular pigment; MPOD = macular pigment optical density.

\section{Mixed Linear Model Effects}

A mixed linear model analysis was carried out on normalized macular pigment optical density versus demographic, behavioral, anthropometric, and plasma biomarker as independent variables. Low education, current smoking, and elevated waist circumference were significant predictors of macular pigment optical density in this regression model (Table 3 ). None of the plasma biomarkers (high-density lipoprotein, triglyceride-to-high-density lipoprotein ratio, total cholesterol-to-high-density lipoprotein ratio, vitamin D, C-reactive protein, or glycated hemoglobin) were significantly associated with macular pigment optical density in this model ( $P>.05$ for all variables; Table 3 ).

\section{DISCUSSION}

The key finding to emerge from this study is the observation that participants with increased central adiposity, as indicated by an elevated waist circumference, had a significantly lower macular pigment optical density compared with those with ideal measures. Macular pigment was also lower among participants with an altered lipoprotein profile, that is, in participants with raised plasma triglyceride-to-high-density lipoprotein ratio, raised plasma total cholesterol-to-high-density lipoprotein ratio, and low plasma high-density lipoprotein. In addition, male sex, current smoking, and low education status were associated with lower macular pigment optical density, findings that concur with previously reported analyses on a similar cohort. ${ }^{29}$ Elevated waist circumference, current smoking, and low educational attainment persisted as predictors of low macular pigment optical density on mixed linear model analysis, after adjusting for all other covariates.

Consistent with most previous reports, ${ }^{24,29,31}$ macular pigment optical density was, in fact, significantly and negatively associated with all measures of overweight/obesity, including body mass index, waist circumference, waist-to-height ratio, waist-to-hip ratio (Pearson $r ; P<.05$ for all). With cutoff levels applied to classify participants into binary categories (obese or nonobese), ${ }^{38}$ elevated waist circumference emerged as the main anthropometric variable associated with lower levels of macular pigment optical density. Carotenoids, such as lutein and zeaxanthin, are known to accumulate in adipose tissue, and in visceral fat in particular ${ }^{31,45}$; therefore, distribution of body fat is an important consideration. ${ }^{46}$ Higher waist circumference has previously been reported to be the strongest nondietary predictor of low macular pigment optical density, ${ }^{24}$ and findings from the Third National Health and Nutrition Examination Survey revealed that participants with higher waist circumference values were increasingly likely to have hypertension, diabetes, dyslipidemia, and the metabolic syndrome, ${ }^{47}$ metabolic correlates independently associated with lower macular pigment optical density. ${ }^{12,13,27}$

As an indicator of "abdominal" obesity, ${ }^{24,47}$ waist circumference is receiving increased attention as a more clinically relevant measure of adiposity compared with body mass index. Intra-abdominal fat may have different metabolic consequences compared with other patterns of fat distribution or overall adiposity, as it is more biologically active. Intra-abdominal fat is associated with inflammation due to the increased production of adipocytokines. ${ }^{48}$ The associated inflammation becomes chronic; therefore, obesity is now considered a low-grade inflammatory condition, which in turn leads to an increase in oxidative stress. ${ }^{49}$ Chronic inflammation and associated oxidative stress exert a greater demand on antioxidant defenses within the body, defenses that tend to be lower in obese individuals ${ }^{50}$ and in older adults. ${ }^{51}$ Sex differences also exist in relation to how body fat is deposited, ${ }^{52}$ and there is evidence for sex-related differences in the accumulation of carotenoids. ${ }^{53,54}$ Interestingly, we found that macular pigment optical density was $9 \%$ lower in men compared with women $(P=.004)$, possibly explained by the fact that men store more fat in the visceral (abdominal) fat depot, ${ }^{52}$ thereby making these pigments less available to retinal tissue. ${ }^{55,56}$ Thus, lower antioxidant activity may relate not only to lower intake of antioxidants and phytochemical rich foods in older adults (e.g., fruits, vegetables, and legumes) ${ }^{57}$ and reduced activity of endogenous antioxidative enzymes, ${ }^{50,51}$ but also to decreased availability ${ }^{52}$ and increased utilization of these molecules. Collectively, these factors may lead to antioxidant depletion, including lower levels of macular pigment in the eye. ${ }^{2,14}$

Another interesting finding is the observation that macular pigment optical density was significantly lower in participants with raised plasma triglyceride-to-high-density lipoprotein ratio $(>0.87$ $\mathrm{mmol} / \mathrm{L}$ ) and in those with raised plasma total cholesterol-to-highdensity lipoprotein ratio ( $>3.5 \mathrm{mmol} / \mathrm{L})$. Although these relationships did not persist on mixed linear model analysis, the lower macular pigment optical density levels observed among participants with low plasma high-density lipoprotein corroborate previous findings that identified an association between plasma high-density lipoprotein and macular pigment optical density. ${ }^{58}$ Dyslipidemia is often characterized by low levels of high-density lipoprotein, raised triglycerides, and smaller, more atherogenic low-density lipoprotein particles. ${ }^{59}$ Because carotenoids are bound to circulating lipoproteins, the relation between plasma levels of the various lipoproteins and macular pigment optical density is of interest. Evidence suggests that high-density lipoprotein is a major carrier of lutein to the eye. ${ }^{27}$ Furthermore, high-density lipoprotein is a 
TABLE 2. MPOD according to plasma biomarkers in a normal population (normalized MPOD)

\begin{tabular}{|c|c|c|c|c|c|c|}
\hline Variable & $\mathrm{n}$ & Mean (SD) MPOD & 25th & 50th & 75th & $P$ \\
\hline \multicolumn{7}{|l|}{ Plasma biomarkers } \\
\hline \multicolumn{7}{|l|}{$\mathrm{TC}(\mathrm{mmol} / \mathrm{L})$} \\
\hline High $>5.00$ & 1549 & $0.222(0.160)$ & 0.096 & 0.192 & 0.312 & \\
\hline \multicolumn{7}{|l|}{$\mathrm{HDL}(\mathrm{mmol} / \mathrm{L})$} \\
\hline Low $\leq 1.6$ & 1463 & $0.218(0.161)$ & 0.096 & 0.183 & 0.305 & \\
\hline \multicolumn{7}{|l|}{ LDL (mmol/L) } \\
\hline Ideal $\leq 2.6$ & 833 & $0.222(0.157)$ & 0.109 & 0.190 & 0.314 & .97 \\
\hline High $>2.6$ & 1756 & $0.223(0.162)$ & 0.098 & 0.193 & 0.312 & \\
\hline \multicolumn{7}{|l|}{$\mathrm{TG}(\mathrm{mmol} / \mathrm{L})$} \\
\hline Ideal $\leq 3.4$ & 1044 & $0.224(0.159)$ & 0.108 & 0.190 & 0.314 & .72 \\
\hline High $>3.4$ & 1541 & $0.222(0.162)$ & 0.098 & 0.194 & 0.311 & \\
\hline \multicolumn{7}{|l|}{ TG/HDL-C ratio } \\
\hline Ideal $\leq 0.87$ & 1187 & $0.233(0.163)$ & 0.106 & 0.204 & 0.322 & $.003^{*}$ \\
\hline High $>0.87$ & 1402 & $0.214(0.158)$ & 0.095 & 0.182 & 0.304 & \\
\hline \multicolumn{7}{|l|}{ TC/HDL-C ratio } \\
\hline Ideal $\leq 3.5$ & 1483 & $0.229(0.164)$ & 0.105 & 0.200 & 0.319 & $.03^{*}$ \\
\hline High $>3.5$ & 1093 & $0.214(0.156)$ & 0.096 & 0.180 & 0.300 & \\
\hline \multicolumn{7}{|l|}{$\mathrm{HbA}_{1 \mathrm{c}}(\%)$} \\
\hline$\leq 5.00$ & 1206 & $0.221(0.159)$ & 0.101 & 0.188 & 0.309 & .63 \\
\hline$>5.00$ & 1388 & $0.225(0.162)$ & 0.101 & 0.196 & 0.316 & \\
\hline
\end{tabular}

ANOVA was used to check for normalized MPOD differences among plasma biomarkers. $P$ values are reported using MPOD square root transformation (normalized MPOD) and reflect the probability associated with the given F statistic. The following cutoffs were applied to plasma levels: HDL (high risk, $\leq 1.6 \mathrm{mmol} / \mathrm{L}$; low risk, $>1.6 \mathrm{mmol} / \mathrm{L}$ ), LDL (high risk, $>2.6 \mathrm{mmol} / \mathrm{L}$; low risk, $\leq 2.6 \mathrm{mmol} / \mathrm{L}$ ), TC (high risk, $>5.00 \mathrm{mmol} / \mathrm{L}$; low risk, $\leq 5.00 \mathrm{mmol} / \mathrm{L}$ ), TG (high risk, $>1.7 \mathrm{mmol} / \mathrm{L}$; low risk, $\leq 1.7 \mathrm{mmol} / \mathrm{L}$ ), TG/HDL-C ratio (high risk, $>0.87 \mathrm{mmol} / \mathrm{L}$; low risk, $\leq 0.87 \mathrm{mmol} / \mathrm{L}$ ), TC/HDL-C ratio (high risk, $>3.5$ $\mathrm{mmol} / \mathrm{L}$; low risk, $\leq 3.5 \mathrm{mmol} / \mathrm{L}$ ), non-HDL (high risk, $>3.4 \mathrm{mmol} / \mathrm{L}$; low risk, $\leq 3.4 \mathrm{mmol} / \mathrm{L}$ ) as per 2016 European Society of Cardiology/European Atherosclerosis Society guidelines, ${ }^{40}$ plasma vitamin D (25-hydroxyvitamin D) levels (deficient, $\leq 50 \mathrm{nmol} / \mathrm{L}$; sufficient, $>50 \mathrm{nmol} / \mathrm{L}$ ) as per Institute of Medicine vitamin D guidelines, ${ }^{41}$ plasma CRP levels (high risk, $>3.00 \mathrm{mg} / \mathrm{L}$; low risk, $\leq 3.00 \mu \mathrm{gg} / \mathrm{L}$ ), ${ }^{35}$ and $\mathrm{HbA}_{1 \mathrm{c}}$ (high, $>5.00 \%$; ideal, $\leq 5.00 \%(42.1 \mathrm{mmol} /$ mol) as per American Diabetes Association guidelines. ${ }^{42}{ }^{*}$ Significant at the .05 level. $\mathrm{CRP}=\mathrm{C}$-reactive protein; $\mathrm{HbA} \mathrm{Ac}_{1 \mathrm{c}}=$ glycated hemoglobin; $\mathrm{HDL}=$ high-density lipoprotein; LDL = low-density lipoprotein; MPOD = macular pigment optical density; non-HDL = total cholesterol minus high-density lipoprotein; SD = standard deviation; sig. = significance; TC = total cholesterol; TC/HDL-C = total cholesterol-to-high-density lipoprotein cholesterol ratio; $\mathrm{TG}=$ triglyceride; $\mathrm{TG} / \mathrm{HDL}-\mathrm{C}=$ triglyceride to high-density lipoprotein cholesterol ratio.

known ligand for scavenger receptor class B type 1 , and the mechanism of xanthophyll uptake in the retina seems to be entirely scavenger receptor class $B$ type 1 dependent. ${ }^{56}$

Vitamin $D$ deficiency has repeatedly been associated with ocular disease. ${ }^{60,61}$ Of interest, we recently reported that individuals with type 2 diabetes who were vitamin $D$ deficient $(\leq 50 \mathrm{nmol} / \mathrm{L})$ had a significantly lower macular pigment optical density and that vitamin D was a significant positive predictor of macular pigment optical density on multivariate analysis in this study group. ${ }^{62}$ This study, however, suggests that the association between vitamin D status and macular pigment optical density does not persist among older adults who are free of ocular pathology. Furthermore, no relationship was observed between macular pigment optical density and the inflammatory marker C-reactive protein. 


\section{A High Density Lipoprotein (mmol/L)}

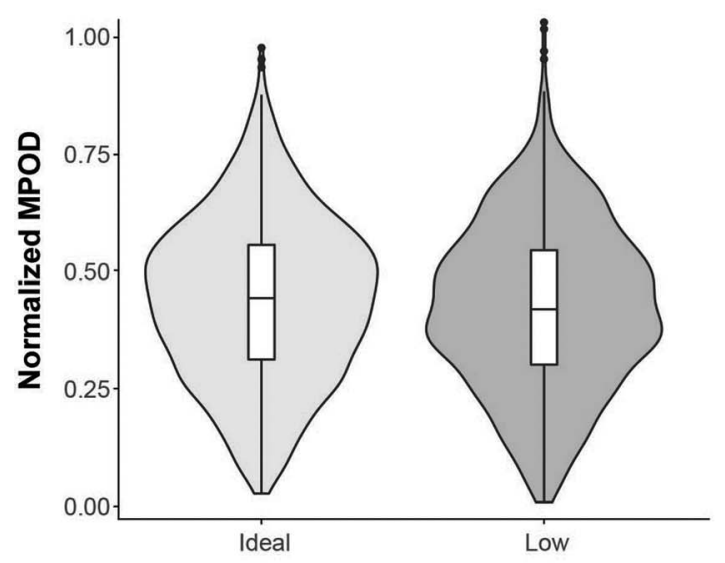

B Triglyceride over High Density Lipoprotein

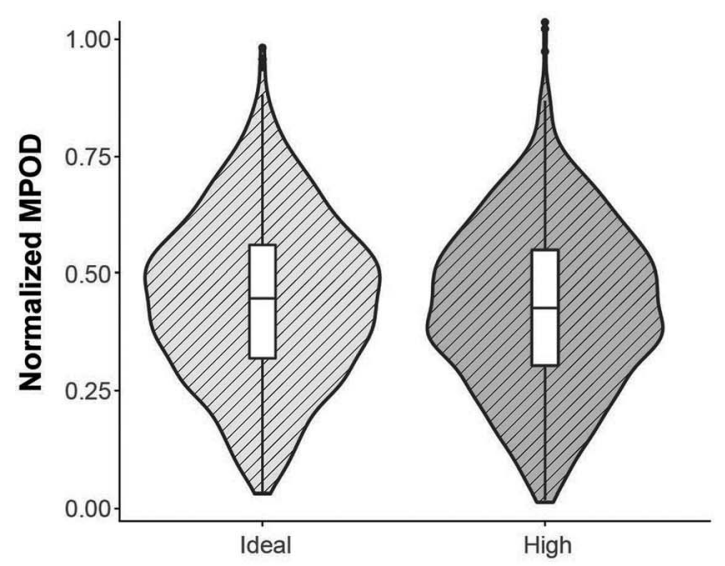

C Total Cholesterol over High Density

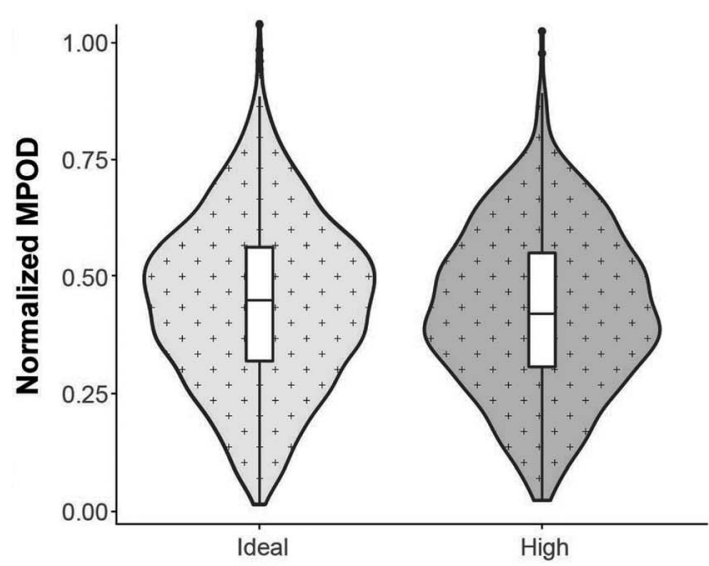

FIGURE 3. Violin plots of MPOD differences illustrating the following: lower MPOD among subjects with low HDL (A), lower MPOD among subjects with raised TG/HDL ratio (B), and lower MPOD among subjects with raised TC/HDL ratio (C; ANOVA). HDL = high-density lipoprotein; $\mathrm{MPOD}=$ macular pigment optical density; $\mathrm{TC} / \mathrm{HDL}=$ total cholesterol-to-high-density lipoprotein ratio; TG/HDL = triglycerideto-high-density lipoprotein ratio.
This population-based representative study advances previously published work that also used The Irish Longitudinal Study on Aging data to explore macular pigment optical density, ${ }^{29}$ as the analyses herein relied much less on self-reported data. A large number of subjects were excluded from the overall macular pigment analysis $(n=2014)$, and possible reasons for this included the following: technical issues, poor fixation, and poor visual acuity, among others. Although customized heterochromatic flicker photometry is one of the most common methods of measuring macular pigment, it is challenging to perform, particularly so for older adults. ${ }^{36}$ Research has shown that some participants have difficulty performing this technique, with dropout rates varying from 10 to $20 \%{ }^{36,63} \mathrm{~A} \mathrm{x}^{2}$ test of independence was performed, however, comparing the center population $(n=5275)$ and the smaller sample of participants with a valid macular pigment optical density assessment ( $n=2594)$, and this test confirmed that there was no significant difference in age, sex, or education status between the two groups. More importantly the attenuated number of participants in the current study did not seem to have an impact on the validity of our findings as we confirmed previously reported analyses on a similar cohort. ${ }^{29}$ Despite differences in inclusion criteria, the relationship between education and smoking status and macular pigment optical density observed therein and elsewhere was confirmed in our analysis. ${ }^{24,29}$ Some discrepancies did emerge, however. Self-reported measures of high cholesterol, for example, were not associated with macular pigment optical density according to the previous analysis, ${ }^{29}$ whereas the analysis of direct cholesterol measures taken herein suggests that dyslipidemia (i.e., low plasma high-density lipoprotein, raised triglyceride-to-high-density lipoprotein ratio, raised total to high-density lipoprotein ratio) may have implications for the delivery and uptake of macular pigment in the eye. In addition, although an inverse association between self-reported high blood pressure and macular pigment was also previously reported, ${ }^{29}$ we found no association between measured hypertension (treated or untreated) and macular pigment optical density on either univariate or mixed linear model analysis. These direct measurements (systolic and diastolic, in millimeters of mercury) would seem to outweigh self-reported data. ${ }^{29}$ Interestingly, we recently reported that individuals with diabetes and hypertension had a significantly lower macular pigment optical density compared with those without, ${ }^{62}$ which suggests that when conditions coexist the redox balance may become increasingly upset, and that oxidative stress may increase considerably in hypertension, especially as a diabetic comorbidity. ${ }^{64}$ Hypertension, however, does not seem to affect or deplete macular pigment, in older adults, who are free of ocular pathology.

A number of variables did not persist as predictors of macular pigment optical density on mixed linear analysis (i.e., sex and plasma lipoproteins) despite significant outcomes on univariate analysis. It is possible that these variables have some underlying associations with other variables. Therefore, when these variables are included in the model, the variance of one is accounted for by the others, and hence, it is no longer significant. Although sex (male) and lipoprotein (low high-density lipoprotein, raised triglyceride to high-density lipoprotein, raised total to high-density lipoprotein ratio) status contribute to lower levels of macular pigment, it seems that waist circumference status is a more robust predictor of low macular pigment optical density. It is important to note, however, that the small difference in mean macular pigment optical density between participants with and without elevated waist circumference suggests that any effect size is likely to 
TABLE 3. Mixed linear model effects: relationship between demographic, behavioral, anthropometric, and plasma biomarkers and MPOD

\begin{tabular}{|c|c|c|c|c|}
\hline Parameter & Estimate & Std. error & $T$ & (Normalized) MPOD $P$ \\
\hline Intercept & 0.448 & 0.085 & 5.297 & $<.0001$ \\
\hline Sex* (female) & 0.011 & 0.008 & 1.284 & .20 \\
\hline \multicolumn{5}{|l|}{ Education } \\
\hline Secondary & 0.023 & 0.010 & 2.376 & .02 \\
\hline Tertiary/higher & 0.038 & 0.010 & 3.866 & $<.0001$ \\
\hline \multicolumn{5}{|l|}{ Smoking† } \\
\hline Past & -0.004 & 0.008 & -0.588 & .56 \\
\hline Current & -0.022 & 0.011 & -2.011 & .04 \\
\hline WC & -0.001 & 0.000 & -2.415 & .02 \\
\hline $\mathrm{HbA}_{1 \mathrm{c}}$ & 0.006 & 0.013 & 0.454 & .65 \\
\hline Vitamin D & -0.000 & 0.000 & -0.728 & .47 \\
\hline $\mathrm{CRP} \ddagger(>3 \mu \mathrm{g} / \mathrm{L})$ & 0.000 & 0.000 & 0.745 & .46 \\
\hline HDL & -0.009 & 0.012 & -0.781 & .44 \\
\hline TG/HDL ratio & -0.001 & 0.005 & -0.161 & .87 \\
\hline TC/HDL ratio & -0.001 & 0.005 & -0.145 & .89 \\
\hline \multicolumn{5}{|l|}{ Hypertension§ } \\
\hline Hypertensive & 0.008 & 0.007 & 1.056 & .29 \\
\hline Hypertensive (not medicated) & 0.002 & 0.010 & 0.188 & .85 \\
\hline Cataracts (no) & 0.018 & 0.014 & 1.291 & .20 \\
\hline \multicolumn{5}{|c|}{ 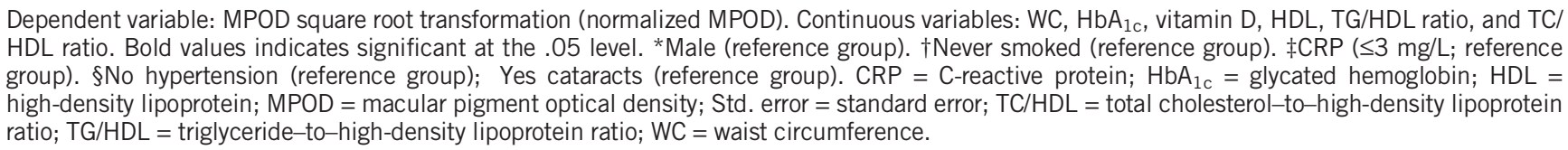 } \\
\hline
\end{tabular}

be modest and not particularly useful in terms of predictive capacity from a clinical perspective.

Macular pigment was measured using customized heterochromatic flicker photometry, a technique that has been validated in older subjects ${ }^{63,65}$ and found to be reliable. ${ }^{63}$ Macular pigment measurements have, however, shown a degree of variability across studies. $^{24,65-67}$ For example, Stringham et al. ${ }^{65}$ measured the density of macular pigment in patients with intermediate AMD using a similar device and retinal eccentricity $\left(0.5^{\circ}\right)$, and the mean optical density was $0.37(0.24)$. Subjects in the Carotenoids in Age-Related Eye Disease Study had average macular pigment levels of $0.36(0.22)$ for the right eye and $0.34(0.21)$ for the left eye, and these participants were of similar age. ${ }^{24}$ We acknowledge that the mean macular pigment optical density in the current study was notably lower than previously reported. ${ }^{24,65}$ Our findings suggest that older Irish adults have lower macular pigment levels on average. It is worth noting that the prevalence of conditions that might affect macular pigment optical density levels is relatively high in Ireland, including AMD, ${ }^{68}$ glaucoma, ${ }^{69}$ and type 2 diabetes. $^{70}$ The lower levels of macular pigment observed in the current study may therefore reflect the combined influence of inadequate dietary intake and increased utilization of these phytonutrients. ${ }^{51}$ Given the variety of mechanisms that may contribute to macular pigment depletion and the ocular risks associated with such depletion in this age group, the importance of diet cannot be overemphasized. The provision of colored fruit and vegetables, which are rich in a broad range of antioxidants including the target carotenoids lutein and zeaxanthin, and/or supplementation with these nutrients represent an obvious recommendation, given the putative protection that this pigment confers against age-related vision loss. These recommendations should now be subject to further research. Limitations of the current study include the failure to assess dietary intake or plasma levels of the carotenoids lutein and zeaxanthin, as macular pigment optical density is influenced by both intake and individual efficacy of absorption. ${ }^{71}$ It is also worth noting that the high variability and lower levels of macular pigment optical density levels across different people may also be subject to genetic variation, ${ }^{72}$ a factor that is not accounted for in our analysis. Information on supplement usage was also not assessed, which would have added to the interpretation of our findings, particularly those in relation to vitamin $D$ and macular pigment optical density. Despite these drawbacks, the representative study design, the unique sampling method (which includes both male and female subjects), the use of measured rather than self-reported biomarkers, ${ }^{29}$ and the detailed exclusion criteria, we believe that our findings and the conclusions drawn from our findings concerning macular pigment optical density are representative of the older Irish population.

In conclusion, we report that elevated waist circumference and dyslipidemia may have important implications for the storage, delivery, and uptake of lutein and zeaxanthin in the eye. Determinants of macular pigment optical density (i.e., education status, tobacco use, waist circumference) remain statistically significant after mixed linear model analysis. Although no strong predictor of macular pigment optical 
density status emerged in this analysis, the capacity of more commonly measured surrogate biomarkers to help identify people at risk of low macular pigment merits further consideration. Given the marginal statistical significance of many of our findings, further research is necessary to refine our understanding of the observed relationships, as macular pigment is not routinely measured in clinical practice.

\section{ARTICLE INFORMATION}

Submitted: February 6, 2020

Accepted: May 25, 2020

Funding/Support: None of the authors have reported funding/support.

Conflict of Interest Disclosure: None of the authors have reported a financial conflict of interest.

Author Contributions: Conceptualization: GS; Data Curation: RAK; Formal Analysis: GS, JSB, JL; Methodology: GS, RAK; Software: JSB, EL; Supervision: GS, DM, JL; Writing - Original Draft: GS; Writing - Review \& Editing: GS, DM, RAK, JL.

\section{REFERENCES}

1. Taylor HR, Keeffe JE. World Blindness: A 21st Century Perspective. Br J Ophthalmol 2001;85:261-6.

2. Lima VC, Rosen RB, Farah M. Macular Pigment in Retinal Health and Disease. Int J Retina Vitreous 2016;2: 19.

3. Siah WF, Loughman J, O'Brien C. Lower Macular Pigment Optical Density in Foveal-involved Glaucoma. Ophthalmology 2015;122:2029-37.

4. Trieschmann M, Van Kuijk FJGM, Alexander R, et al. Macular Pigment in the Human Retina: Histological Evaluation of Localization and Distribution. Eye (Lond) 2008;22:132-7.

5. Snodderly DM, Auran JD, Delori FC. The Macular Pigment. II. Spatial Distribution in Primate Retinas. Invest Ophthalmol Vis Sci 1984;25:674-85.

6. Khachik F, Bernstein PS, Garland DL. Identification of Lutein and Zeaxanthin Oxidation Products in Human and Monkey Retinas. Invest Ophthalmol Vis Sci 1997; 38:1802-11.

7. Loughman J, Davison PA, Nolan JM, et al. Macular Pigment and Its Contribution to Visual Performance and Experience. J Optom 2010;3:74-90.

8. Age-Related Eye Disease Study 2 Research Group. Lutein + Zeaxanthin and Omega-3 Fatty Acids for Age-related Macular Degeneration: The Age-Related Eye Disease Study 2 (AREDS2) Randomized Clinical Trial. JAMA 2013;309:2005-15.

9. Hu BJ, Hu YN, Lin S, et al. Application of Lutein and Zeaxanthin in Nonproliferative Diabetic Retinopathy. Int J Ophthalmol 2011;4:303-6.

10. Chous AP, Richer SP, Gerson JD, et al. The Diabetes Visual Function Supplement Study (DiVFuSS). Br J Ophthalmol 2016;100:227-34.

11. Beatty S, Murray IJ, Henson DB, et al. Macular Pig ment and Risk for Age-related Macular Degeneration in Subjects from a Northern European Population. Invest Ophthalmol Vis Sci 2001;42:439-46.

12. Lima VC, Rosen RB, Maia M, et al. Macular Pigment Optical Density Measured by Dual-wavelength Autofluorescence Imaging in Diabetic and Nondiabetic Patients: A Comparative Study. Invest Ophthalmol Vis Sci 2010;51:5840-5.
13. Scanlon G, Connell P, Ratzlaff M, et al. Macular Pigment Optical Density Is Lower in Type 2 Diabetes, Compared with Type 1 Diabetes and Normal Controls. Retina 2015;35:1808-16

14. Beatty S, Koh HH, Phil M, et al. The Role of Oxidative Stress in the Pathogenesis of Age-related Macular Degeneration. Surv Ophthalmol 2000;45:115-34.

15. Kowluru RA, Chan PS. Oxidative Stress and Diabetic Retinopathy. Exp Diabetes Res 2007;2007:43603.

16. Hollyfield JG. Age-related Macular Degeneration: The Molecular Link between Oxidative Damage, Tissue-specific Inflammation and Outer Retinal Disease: The Proctor Lecture. Invest Ophthalmol Vis Sci 2010;51:1275-81.

17. Wellen KE, Hotamisligil GS. Inflammation, Stress, and Diabetes. J Clin Invest 2005;115:1111-9.

18. Ozawa Y, Sasaki M, Takahashi N, et al. Neuroprotective Effects of Lutein in the Retina. Curr Pharm Des 2012;18:51-6.

19. Xue C, Rosen R, Jordan A, et al. Management of Ocular Diseases Using Lutein and Zeaxanthin: What Have We Learned from Experimental Animal Studies? J Ophthalmol 2015;2015:523027.

20. Chao SC, Vagaggini T, Nien CW, et al. Effects of Lutein and Zeaxanthin on LPS-induced Secretion of IL-8 by Uveal Melanocytes and Relevant Signal Pathways. J Ophthalmol 2015;2015:152854.

21. Loughman J, Scanlon G, Nolan JM, et al. An Evaluation of a Novel Instrument for Measuring Macular Pigment Optical Density: The MPS 9000. Acta Ophthalmol 2012;90:e90-7.

22. Hammond BR, Jr., Curran Celentano J, Judd S et al. Sex Differences in Macular Pigment Optical Density: Relation to Plasma Carotenoid Concentrations and Dietary Patterns. Vision Res 1996;36:2001-12.

23. Hammond BR, Jr., Fuld K, Snodderly MD. Iris Color and Macular Pigment Optical Density. Exp Eye Res 1996;62:293-7.

24. Mares JA, LaRowe TL, Snodderly DM, et al. Predictors of Optical Density of Lutein and Zeaxanthin in Retinas of Older Women in the Carotenoids in Age-Related Eye Disease Study, an Ancillary Study of the Women's Health Initiative. Am J Clin Nutr 2006;84:1107-22.

25. Olmedilla Alonso B, Beltrán-de-Miguel B, EstévezSantiago R, et al. Markers of Lutein and Zeaxanthin Status in Two Age Groups of Men and Women: Dietary Intake, Serum Concentrations, Lipid Profile and Macular Pigment Optical Density. Nutr J 2014;13:52.

26. Hammond BR, Jr., Johnson EJ, Russell RM, et al. Dietary Modification of Human Macular Pigment Density. Invest Ophthalmol Vis Sci 1997;38:1795-801.

27. Connor WE, Duell PB, Kean R, et al. The Prime Role of $\mathrm{HDL}$ to Transport Lutein into the Retina: Evidence from HDL-deficient WHAM Chicks Having a Mutant ABCA1 Transporter. Invest Ophthalmol Vis Sci 2007; 48:4226-31

28. Loane E, Nolan JM, Beatty S. The Respective Relationships between Lipoprotein Profile, Macular Pig ment Optical Density, and Serum Concentrations of Lutein and Zeaxanthin. Invest Ophthalmol Vis Sci 2010 51:5897-905.
29. Nolan JM, Feeney J, Kenny RA, et al. Education Is Positively Associated with Macular Pigment: The Irish Longitudinal Study on Ageing (TILDA). Invest Ophthalmol Vis Sci 2012;53:7855-61.

30. Bovier ER, Lewis RD, Hammond BR, Jr. The Relationship between Lutein and Zeaxanthin Status and Body Fat. Nutrients 2013;5:750-7.

31. Hammond BR, Jr., Ciulla TA, Snodderly DM. Macular Pigment Density Is Reduced in Obese Subjects. Invest Ophthalmol Vis Sci 2002;43:47-50.

32. Kearney PM, Cronin H, O'Regan C, et al. Cohort Profile: The Irish Longitudinal Study on Ageing. Int J Epidemiol 2011;40:877-84.

33. Donoghue OA, McGarrigle CA, Foley M, et al. Cohort Profile Update: The Irish Longitudinal Study on Ageing (TILDA). Int J Epidemiol 2018;47:1398-1398I.

34. Kenny RA, Whelan BJ, Cronin $\mathrm{H}$, et al. The Design of The Irish Longitudinal Study on Ageing. 2010. Available at: https://tilda.tcd.ie/publications/reports/pdf/Report DesignReport.pdf. Accessed September 24, 2019.

35. Cronin H, O'Regan C, Finucane C, et al. Health and Aging: Development of The Irish Longitudinal Study on Ageing Health Assessment. J Am Geriatr Soc 2013;61 (Suppl. 2):S269-78.

36. Nolan JM, Kenny R, O'Regan C, et al. Macular Pigment Optical Density in an Ageing Irish Population: The Irish Longitudinal Study on Ageing. Ophthalmic Res 2010;44:131-9.

37. Sikaris KA. The Clinical Biochemistry of Obesity. Clin Biochem Rev 2004;25:165-81.

38. World Health Organization. Waist Circumference and Waist-hip Ratio: Report of a WHO Expert Consultation, Geneva, 8-11 December 2008. 2011. Available at: http:// whqlibdoc.who.int/publications/2011/9789241501491 eng.pdf. Accessed October 13, 2019.

39. Ashwell M, Gibson S. A Proposal for a Primary Screening Tool: Keep Your Waist Circumference to Less than Half Your Height. BMC Med 2014;12:207.

40. Catapano AL, Graham I, De Backer G, et al. 2016 ESC/EAS Guidelines for the Management of Dyslipidaemias. Eur Heart J 2016;37:2999-58.

41. Ross AC, Manson JE, Abrams SA, et al. The 2011 Report on Dietary Reference Intakes for Calcium and Vitamin D from the Institute of Medicine: What Clinicians Need to Know. J Clin Endocrinol Metab 2011;96:53-8.

42. Koenig W, Sund M, Fröhlich M, et al. C-reactive Protein, a Sensitive Marker of Inflammation, Predicts Future Risk of Coronary Heart Disease in Initially Healthy Middle-aged Men: Results from the MONICA (Monitoring Trends and Determinants in Cardiovascular Disease) Augsburg Cohort Study, 1984 to 1992. Circulation 1999;99:237-42

43. Barrett $A$, Burke $H$, Cronin $H$, et al. Fifty Plus in Ireland 2011: First Results from The Irish Longitudinal Study on Ageing (TILDA). 2011. Available at: https://epubs.rcsi.ie/ psycholrep/45.pdf. Accessed October 13, 2019

44. American Diabetes Association. 2. Classification and Diagnosis of Diabetes. Diabetes Care 2015;38:S8-16.

45. Johnson EJ, Neuringer M, Russell RM, et al. Nutritional Manipulation of Primate Retinas, III: Effects of 
Lutein or Zeaxanthin Supplementation on Adipose Tissue and Retina of Xanthophyll-free Monkeys. Invest Ophthalmol Vis Sci 2005;46:692-702

46. Chung HY, Ferreira ALA, Epstein S, et al. Sitespecific Concentrations of Carotenoids in Adipose Tissue: Relations with Dietary and Serum Carotenoid Concentrations in Healthy Adults. Am J Clin Nutr 2009; 90:533-9.

47. Janssen I, Katzmarzyk PT, Ross R. Body Mass Index, Waist Circumference, and Health Risk: Evidence in Support of Current National Institutes of Health Guidelines. Arch Interm Med 2002;162:2074-9.

48. Kwon H, Pessin JE. Adipokines Mediate Inflammation and Insulin Resistance. Front Endocrinol (Lausanne) 2013;4:71.

49. Fernández Sánchez A, Madrigal Santillán E, Bautista M, et al. Inflammation, Oxidative Stress, and Obesity. Int J Mol Sci 2011;12:3117-32.

50. Savini I, Catani MV, Evangelista D, et al. Obesityassociated Oxidative Stress: Strategies Finalized to Improve Redox State. Int J Mol Sci 2013;14:10497-538.

51. Morales-Gonzalez JA, ed. Oxidative Stress and Chronic Degenerative Diseases - A Role for Antioxidants. BoD-Books on Demand; May 22, 2013.

52. Blaak E. Gender Differences in Fat Metabolism. Curr Opin Clin Nutr Metab Care 2001;4:499-502.

53. Nolan J, O'Donovan O, Kavanagh H, et al. Macular Pigment and Percentage of Body Fat. Invest Ophthalmol Vis Sci 2004;45:3940-50.

54. Johnson EJ, Hammond BR, Yeum KJ, et al. Relation among Serum and Tissue Concentrations of Lutein and Zeaxanthin and Macular Pigment Density. Am J Clin Nutr 2000;71:1555-62.

55. Gruber M, Chappell R, Millen A, et al. Correlates of Serum Lutein + Zeaxanthin: Findings from the Third
National Health and Nutrition Examination Survey. J Nutr 2004;134:2387-94.

56. During A, Doraiswamy S, Harrison EH. Xanthophylls Are Preferentially Taken up Compared with $\beta$-carotene by Retinal Cells Via a SRBI-dependent Mechanism. J Lipid Res 2008;49:1715-24.

57. O'Connor D, Leahy S, McGarrigle C. Consumption Patterns and Adherence to the Food Pyramid. Health and Wellbeing: Active Ageing for Older Adults in Ireland. 2017:50. Available at: https://tilda.tcd.ie/ publications/reports/pdf/w3-key-findings-report/Chapter \%204.pdf. Accessed October 13, 2019.

58. Renzi LM, Hammond BR, Jr., Dengler M, et al. The Relation between Serum Lipids and Lutein and Zeaxanthin in the Serum and Retina: Results from Crosssectional, Case-control and Case Study Designs. Lipids Health Dis 2012;11:33.

59. Krauss RM, Siri PW. Metabolic Abnormalities: Triglyceride and Low-density Lipoprotein. Endocrinol Metab Clin 2004;33:405-15.

60. Millen AE, Sahli MW, Nie J, et al. Adequate Vitamin D Status Is Associated with the Reduced Odds of Prevalent Diabetic Retinopathy in African Americans and Caucasians. Cardiovasc Diabetol 2016;15:128.

61. Berridge MJ. Vitamin D Deficiency and Diabetes. Biochem J 2017;474:1321-32.

62. Scanlon G, McCartney D, Butler JS, et al. Identification of Surrogate Biomarkers for the Prediction of $\mathrm{Pa}$ tients at Risk of Low Macular Pigment in Type 2 Diabetes. Curr Eye Res 2019;44:1369-80.

63. Snodderly DM, Mares JA, Wooten BR, et al. Macular Pigment Measurement by Heterochromatic Flicker Photometry in Older Subjects: The Carotenoids and Age-related Eye Disease Study. Invest Ophthalmol Vis Sci 2004;45:531-8.
64. Pouvreau C, Dayre A, Butkowski EG, et al. Inflammation and Oxidative Stress Markers in Diabetes and Hypertension. J Inflamm Res 2018;11:61-8.

65. Stringham JM, Hammond BR, Nolan JM, et al. The Utility of Using Customized Heterochromatic Flicker Photometry (cHFP) to Measure Macular Pigment in Patients with Age-related Macular Degeneration. Exp Eye Res 2008;87:445-53.

66. Iannaccone A, Mura M, Gallaher KT, et al. Macular Pigment Optical Density in the Elderly: Findings in a Large Biracial Midsouth Population Sample. Invest Ophthalmol Vis Sci 2007;48:1458-65.

67. Ciulla TA, Hammond BR, Jr. Macular Pigment Density and Aging, Assessed in the Normal Elderly and Those with Cataracts and Age-related Macular Degeneration. Am J Ophthalmol 2004;138:582-7.

68. Akuffo KO, Nolan J, Stack J, et al. Prevalence of Age-related Macular Degeneration in the Republic of Ireland. Br J Ophthalmol 2015;99:1037-44.

69. Coffey M, Reidy A, Wormald R, et al. Prevalence of Glaucoma in the West of Ireland. $\mathrm{Br} \mathrm{J}$ Ophthalmol 1993;77:17-21.

70. Leahy S, O'Halloran AM, O'Leary N, et al. Prevalence and Correlates of Diagnosed and Undiagnosed Type 2 Diabetes Mellitus and Pre-diabetes in Older Adults: Findings from The Irish Longitudinal Study on Ageing (TILDA). Diabetes Res Clin Pract 2015; 110:241-9.

71. Schupp C, Olano Martin E, Gerth C, et al. Lutein, Zeaxanthin, Macular Pigment, and Visual Function in Adult Cystic Fibrosis Patients. Am J Clin Nutr 2004; 79:1045-52.

72. Meyers KJ, Johnson EJ, Bernstein PS, et al. Genetic Determinants of Macular Pigments in Women of the Carotenoids in Age-related Eye Disease Study. Invest Ophthalmol Vis Sci 2013;54:2333-45. 